EDITORIAL

\title{
THERAPEUTIC TOOLS FOR REPRESENTING SUBJECTIVE REALITY: CHANGING THE PERCEIVED PAST, PRESENT, AND FUTURE
}

Postmodernism is an intellectual statement about cultural and historical realities and how they can be challenged by other viewpoints. In literature and related disciplines in which interpretation is a central concern, it has garnered wide application. In behavioural science and practice, application of the construct is more challenging insofar as problems do not disappear upon acquiring a semantic case of object impermanence. Yet the conceptualisation of behaviour in terms of trait-situation, for example, does suggest that certain aspects of behaviour are characteristic of the person and other aspects are supported by the setting. Hence, "seeing things differently" may reinvent some human problems in which previous or present habits of thinking and behaving pose a threat to development and future functioning.

Most working psychologists undertake with their clients a cognitive-behavioural problem-solving process that puts in place more functional thoughts and ways to cope with real or imagined problems and to attribute responsibility for implementing that change. Comparative therapeutic evaluations and empirical identification of mechanisms underlying change when it occurs are required.

Activation of the client's subjective view of the problem and its solution is providing some alternative problemsolving strategies that can enhance the therapeutic repertoire. Some of these frameworks for practice are examined in this issue. Because subjectivity necessarily raises confronting issues for the therapist such as supervision, selfawareness, and self-analysis, the place of these methodologies in the canon, such as it is, must be established carefully. Instead of an eclectic drifting style of "sweet talk" supported by therapist confidence and sociable interactions with the client, these subjective approaches to working with child and adolescent clients need to be structured and validated by the normal processes of evaluation, protocols, documentation of goals for accountability, and disciplined reflection about practice. On the other hand, modal clinical practice often evokes adapted methodologies.

The three authors in this collection show what may be seen by looking beyond the client's presenting problem and by reinventing the past or future. Yet the colleagial gaze on professional conversations is invited by these authors, and the need for sensitivity to the limits and issues involved in these therapeutic conversations is acknowledged. The generation of stories does not serve to promote self-deluding explanations and choices about a current predicament by a client.

Three different proposals are made for conceptualising the client's subjective view and seeking a more adaptive connection between past, present, and future. These accounts of the change tools of these therapies for seeing things differently can be compared with existing tools. For example, the types and aims of constructive questioning (e.g., externalising between "me and my problem" in narrative therapy; behavioural exceptions and the miracle question for when behaviour is different in solution-focused brief therapy) and the psychoanalytic processing of transference and countertransference material (e.g., therapist nondisclosure and avoidance of magical intrusion by the perceptive therapist) may help practitioners to reflect on their own practice when working with young clients. 
...cont'd from page 1

Social constructivism is the model underlying two of the papers, although their bases differ markedly. One is a long "script-writing" process between therapist-counsellor and client. The other is a relatively brief school-based intervention process in which an indirect collaborative group of experienced teachers mediates the therapist-child relationship. Both of these constructivist approaches to therapy are focused on the desired future. Broadly, behavioural operationalisation of individualised goals for therapy proceeds directly to "how do you want to be?" without detailed assessment of what is wrong now. Problems restricting current actions are separated from the person, and goals for future conduct and feeling are specified. Such strategies appear to affect the commitment of the client or group of clients (e.g., teachers of a child) to behavioural change, to increase attention to behaviours likely to achieve change, and to transfer responsibility for implementing that change to the client. Comparative therapeutic evaluations and empirical identification of mechanisms underlying change when it occurs are required.

In the third, psychodynamic paper, the student looking backwards to the past may be repeating inappropriate ways of interaction. In this approach, talk rather than action is proposed to detoxify bad feelings. However, a central concern of the emotional exchange in this approach is not to overwhelm the adolescents's fragile boundaries. The strategy is to strengthen the inner working model by creating a safe "containing" relationship between past and future.

In some respects, the arguments in these papers are quite different from each other and from the established behavioural and cognitive methodologies. However, their respective explorations of aspects of the therapist-client relationship share a common concern to strengthen the client.
Whether by narrative excavation of a preferred future reality, active team support for desirable outcomes, or supporting boundaries of the client, these therapies validate, respect, and permit the client to attend to and interpret their experience.

\section{REFERENCES}

Bryer, F. (1994, July). 101 developmental psychologists: Preliminary survey results. Poster presented at the 8th Australasian Developmental Conference, Melbourne.

Kazdin, A. E., \& Weisz, J. R. (1998). Identifying and developing empirically supported child and adolescent treatments. Journal of Consulting and Clinical Psychology, 66 (1), 19-36.

\section{NOTICES}

Intergenerational education programs networking. Contact Heather Hill, (03) 9730 1248, 1 King St., Yarra Glen, 3775

Educational psychologist: Expanding Bairnsdale country practice. Contact Manager (03) 51524777 , fax (03) 51523457

Special issue: Longitudinal studies in Australasia (closing May 1998). Index entries of nature and statistics of project or brief reports on project findings and publications are invited. Contact Editor.

Special issue: Developmental language difficulties (November, 1998). Contact Guest Editors: Felicity Cronan (03-9899 8859) and Jan Fletcher (09-380 3275, jan@psy.uwa.edu.au 